\title{
Inadvertently transected left superior pulmonary vein during thoracoscopic left lower lobectomy
}

\author{
Yangki Seok and Eungbae Lee
}

\begin{abstract} in pulmonary lobectomy. into stump of divided common trunk via thoracotomy. from complications.

Keywords: Anatomical anomaly, Pulmonary vein, Lung cancer surgery

\section{Background}

Pulmonary vascular variations are significant in thoracic surgery as such vascular anomalies can present a number of surgical complications [1]. Dividing a pulmonary vein that should be preserved can lead to potentially life-threatening complications [2]. In this report we describe a case where we inadvertently divided the left superior pulmonary vein (LSPV) during thoracoscopic left lower lobectomy in a lung cancer patient.
\end{abstract}

Background: There are several anatomical variations of the pulmonary vein which can cause serious complications

Case presentation: We inadvertently divided the left superior pulmonary vein during thoracoscopic left lower lobectomy in a lung cancer patient. Retrospective review of the preoperative computed tomography showed extra-pericardial common trunk of the left pulmonary venous system. Left superior pulmonary vein was reimplanted

Conclusions: Awareness of vascular anomalies will help thoracic surgeons to prevent potential morbidity and mortality

\section{Case presentation}

The chest computed tomography (CT) of a 74-year-old female showed a lobulated cancer in the lateral basal segment of the left lower lobe measuring $1.5 \mathrm{~cm}$ in diameter, for which the patient underwent thoracoscopic left lower lobectomy. During this operation, the pulmonary vein was initially divided using a $2.5-\mathrm{mm}$ stapling device (Echelon Flex 60 Endopath Stapler, Ethicon Endo-Surgery, LLC, Guaynabo, Puerto Rico 00969 USA). Next, the common basal and the superior segmental arteries were divided simultaneously by employing the same

\footnotetext{
* Correspondence: bay@knu.ac.kr

Department of Thoracic and Cardiovascular Surgery, Kyungpook National University Medical Center, 807 Hogukno, Buk-gu, Daegu 41404, Republic of Korea
}

device, then the left lower lobar bronchus was divided using a 4.1-mm stapling device (Echelon Flex 60 Endopath Stapler). During post-lobectomy exploration, the LSPV was not be visualized in the expected location. Retrospective review of the preoperative chest CT showed anatomical variation in the pulmonary venous system. In this case, the superior and inferior pulmonary veins had formed a common trunk outside the pericardium and drained into the left atrium (Fig. 1). After heparin injection, all staples on the LSPV and an half of the aples on the common trunk were removed under tra-pericardial partial clamping of left atrium (LA) via thacotomy. The patient became bradycardic during the LA. It was difficult the LA without causing bradycardia, and the optinuous 4-0 Prolene sutures. The LSPV was reimplanted in the location of the left inferior pulmonary vein in the stump of the divided common trunk, rather than its original site. This was because the length of the original site of the LSPV was shortened due to the stapling direction at the time of pulmonary vein division, and the length for reimplantation could not be approximated. The patient 


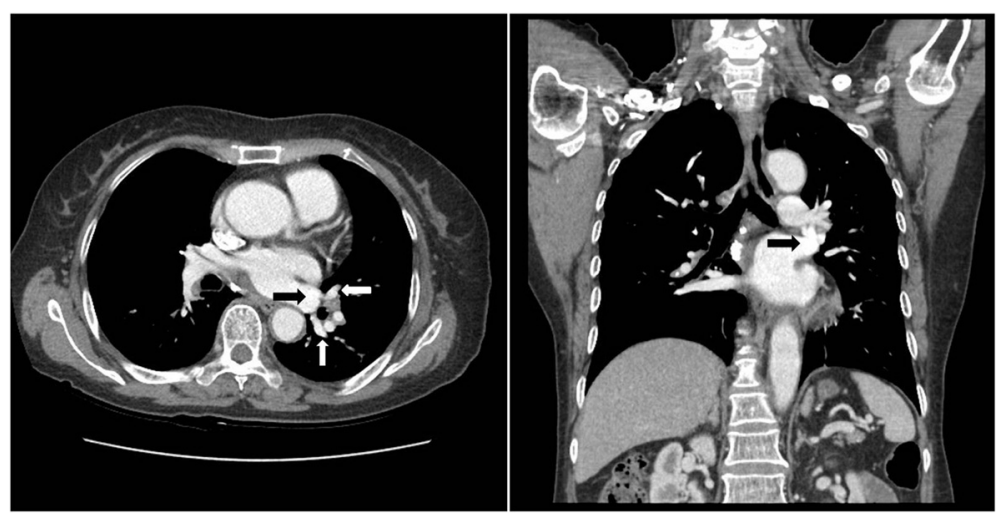

Fig. 1 Preoperative chest computed tomography showed that superior and inferior pulmonary veins (white arrow) had formed a extrapericardial common trunk (black arrow)

recovered well from the procedure and was transferred to the general ward, and was discharged 6 days after the operation without any complications.

\section{Discussion}

Pulmonary vascular anomalies are significant in thoracic surgery [1]. Thoracic surgeons tend to focus more on variations of the pulmonary arteries than the pulmonary veins during preoperative evaluation because the majority of severe intra-operative complications during pulmonary lobectomy are related to the injury of major pulmonary arteries [3]. However, pulmonary venous anatomical variations are more common than those of pulmonary arterial branches [4]. Ligation of a pulmonary vein that should be preserved can lead to severe pulmonary edema, which may cause potentially life-threatening complications such as infection and respiratory distress [2]. Disruption of the pulmonary vein can also lead to complicated and longer procedures [5].

In consideration of the drainage pattern of the left pulmonary vein, the incidence of the common trunk forming one ostium in the LA is $14 \%$ [6]. This can be divided into two types: the first type where the common trunk vein is less than $1 \mathrm{~cm}$ long, and the other where it is more than $1 \mathrm{~cm}$ long that drains into the LA. The incidence of having a common trunk that is longer than $1 \mathrm{~cm}$ is $3.5 \%$ [6]. In this case, the superior and inferior pulmonary veins formed an extra-pericardial common trunk which was longer than $1 \mathrm{~cm}$. The LSPV was reimplanted after lower lobectomy, because we had not confirmed the exact vascular anatomy and divided the common trunk consisting of the superior vein and the inferior vein, mistaking it as the inferior pulmonary vein. This is because of the surgeons' tendency to only visualize the local surgical field rather than checking the general vascular anatomy around the operation site in thoracoscopic surgery. Retrospective review of the preoperative CT showed that the superior and inferior pulmonary veins joined to form the common trunk, which suggests that preoperative diagnosis of this variation is quite possible.

\section{Conclusion}

Awareness of vascular anomalies is very important in excising the pulmonary lobe for lung cancer. Especially closer attention is required in thoracoscopic procedures as the surgical view is more limited than in a thoracotomy. Keeping such vascular anomalies in mind will help thoracic surgeons to prevent potential morbidity and mortality from complications.

\section{Abbreviations}

$C T$ : computed tomography; LA: left atrium; LSPV: left superior pulmonary vein.

\section{Authors' contributions}

All authors performed the operation. YS prepared the manuscript. EL edited manuscript. Both authors read and approved the final manuscript.

\section{Competing interests}

The authors declare that they have no competing interests.

\section{Consent}

Written informed consent was obtained from the patient for the publication of this report and any accompanying images.

Received: 17 January 2016 Accepted: 17 April 2016

Published online: 26 May 2016

\section{References}

1. Yoldas B, Gursoy S. A pulmonary vascular variation to be considered in resective lung surgical procedures. Ann Thorac Surg. 2014;97:715.

2. Sugimoto S, Izumiyama O, Yamashita A, Baba M, Hasegawa T. Anatomy of inferior pulmonary vein should be clarified in lower lobectomy. Ann Thorac Surg. 1998;66:1799-800.

3. Liang C, Wen H, Guo Y, Shi B, Tian Y, Song Z, et al. Severe intraoperative complications during VATS Lobectomy compared with thoracotomy lobectomy for early stage non-small cell lung cancer. J Thorac Dis. 2013;5:513-7.

4. Rajiah P, Kanne JP. Computed tomography of pulmonary venous variants and anomalies. J Cardiovasc Comput Tomogr. 2010;4:155-63.

5. Matsumoto I, Ohta Y, Tsunezuka Y, Sawa S, Fujii S, Saito K, et al. A surgical case of lung cancer in a patient with the left superior and inferior pulmonary veins forming a common trunk. Ann Thorac Cardiovasc Surg. 2005;11:316-9.

6. Marom EM, Herndon JE, Kim YH, McAdams HP. Variations in pulmonary venous drainage to the left atrium: implications for radiofrequency ablation. Radiology. 2004;230:824-9. 